\title{
The topical collection on quality criteria for futures research: a short introduction
}

\author{
Osmo Kuusi ${ }^{1} \cdot$ Kerstin Cuhls $^{2} \cdot$ Karlheinz Steinmüller ${ }^{3}$
}

Received: 21 November 2015 / Accepted: 23 November 2015 /Published online: 19 December 2015

(C) The Author(s) 2015. This article is published with open access at Springerlink.com

\section{Background}

During recent years, questions of quality and good practice gained more traction in the European futures studies community. For this reason, the organizers of the international conference "Futures Studies Tackling Wicked Problems", held in Turku, Finland, in June 2015, decided to devote one of the fifteen thematic sessions to methodological issues with a special focus on quality. This session was jointly organized and chaired by the authors of this introduction. It comprised six sub sessions with a total of twenty papers presented: introduction, critical assessments of futures research methodology, methodological challenges of participatory futures research, methods for security focused futures research, methodological challenges in business foresight and methodological challenges of regional differences.

The common motivation behind the methodology sessions was to debate the state of the art of futures studies, standards for good research and quality criteria to evaluate scientific futures research. One of the related aims was to discuss the differences between scientific futures research and practically oriented or consultative futures work with respect to quality issues. Although the organizers of the methodology sessions were aware

This article is part of the Topical Collection "Quality Criteria for Futures Research"

Osmo Kuusi

osmo.kuusi@utu.fi

1 Aalto University, Turku University, Helsinki, Finland

2 Fraunhofer Institute for Systems and Innovation Research ISI, Karlsruhe, Germany

3 Foresigt Company Z_punkt, Berlin, Germany that futures researchers use and further develop methods from other branches of research, a special aim of the sessions was to discuss basic concepts and approaches that would provide the basis for a specific scientific "paradigm" or frame of futures research. For that purpose, the organizers wrote an "anchor article" [14] that the magazine Futura published in spring 2015. The speakers of the methodology sessions were invited to read the Futura article prior to the Conference, and it was discussed in the first introductory methodology session. The speakers were asked to comment the article in their presentations or link their research to it. The majority of the speakers also commented the article.

This topical collection contains a selection of papers discussed during the Turku conference. Most of them were adapted to integrate comments and feedback during and after the conference. Some papers had to be left out because of publication deadlines and volume restrictions. Nevertheless, this collection covers rather all relevant quality issues scrutinized during the conference.

\section{Articles of the topical collection}

The collection starts with a new version of the 'anchor article' [15] that differs in several points from the old one [14]. The main point is a new, and, as we hope, improved definition of the six quality criteria related to the suggested Futures Map frame. Besides that, we put the emphasis less on general epistemological issues, but tried to discuss and compare different perspectives on quality more in depth.

The 'anchor article' suggests that the Futures Map is a promising frame for scientific futures research. As will be discussed, below, the concept "future(s) map" is not new. Many futurists have used the map metaphor. Some have used very similar framing concepts though they have not called it 
the future(s) map. First of all, this concerns various scenario approaches that are compared with the Futures Map [15]. In his remarks and commentaries, Osmo Kuusi motivates and illustrates the Futures Map frame and the quality criteria defined within that frame. He also comments other articles of the Topical Collection and presents some ideas concerning the ways that might improve validity evaluations based on the suggested Futures Map frame.

The papers in this Topical Collection discuss quality criteria of futures research or foresight from different points of view. It is possible to characterize these perspectives using the main categories of the quality criteria suggested in [15]. On the one hand, quality criteria might concern (1) futures research or foresight process (internal validity), (2) its results (external validity of the Futures Map) or (3) the use of the results (the use of the Futures Map). On the other hand, you can discuss quality criteria on different levels: (a) on a basic philosophy level, (b) on the level of pragmatic general criteria and (c) on a special context level.

The paper of Anna Kononiuk and Anna Sacio-Szymańska [9] discusses quality criteria of foresight in the business environment focusing on the poorest part of Poland: Podlaskie region. The quality criteria discussed in the article concern mostly foresight process in the special business context (1c). The focus of the interest of the authors was also the use of foresight results in companies (3c).

First of all, the study by Kononiuk and Sacio [9] describes how foresight is used in Podlaskie region companies. Further on, it discusses the actual or possible benefits of foresight for the companies. Based on the results of an empirical study of Rohrbeck and Schwartz [21], the authors conclude that the formalized process of foresight might increase the ability of Podlaskie companies in detection, interpretation and reaction to unprecedented events and trends. Foresight might also improve the ability of organizational learning.

Only large companies in the Podlaskie region have some foresight activities. Just this group of companies realized that the identification of trends and drivers might have considerable impact on a company's strategy in the long term. SMEs in the Podlaskie region do not plan many years ahead, they focus instead on identifying short-term trends, setting short-term objectives and building short-term plans. Entrepreneurs of small and medium size companies prefer to work on concrete products, technologies and market needs regarding futures research as marked by rapid change.

The authors conclude that the focus on organizational learning based on employee empowerment is the most important prerequisite (or the quality criterion) of active involvement of enterprises into foresight in the Podlaskie region. The authors perceive business coaching as techniques, which would be instrumental to introducing a high-impact learning culture model and to attain the desired level of foresight maturity. According to the authors, the advantages of that coaching method are increasing effectiveness of entire companies, optimization of decisionmaking process and more effective change management. Quality in this regard is not measured by the results of foresight studies in terms of a Futures Map, but by its impact on organizational culture.

The article of Vasamo [24] is the other article that discusses quality criteria in a specified context. The context is the Radical Technology Inquirer (RTI). It is a tool developed in the Committee for the Future of the Finnish Parliament for technology foresight that effectively takes into account the impacts of emerging radical technologies.

Vasamo uses systematically both the internal and pragmatic external validity criteria of the Futures Map suggested by [15]. Her article shows that the pragmatic generic quality criteria of [15] function well also in her specified context. Using the above classification, her quality criteria belong to the classes' $1 \mathrm{~b}$ and $2 \mathrm{~b}$. The systematic use of the quality criteria shown in [15] is possible because the RTI is a continuously developing Futures Map including now 20 Global Value Producing Networks (GVPNs) and hundred most promising Radical Technological Solutions.

The articles of Kosow [10] and Shala [23] do not have specified application contexts. Shala's article discusses quality criteria on the basic philosophy level. According to her, regardless of the perception of the aims of science, a crucial characteristic of all forms of scientific inquiry is objectivity. The claim of objectivity responds to the idea that science should rely upon facts rather than wishes. The objectivity requirement is especially important during the building process of the Futures Map. In that way, Shala discusses the quality criteria first of all on the level 1a.

Referring to Longino [16], Shala considers that science can provide two different forms of objectivity. First, there is the objectivity that is bound up with questions about the truth and referential character of scientific theories. This is the basic approach of scientific realism. As objectivity in the positivist understanding is limited to rejecting or accepting hypotheses based on observational and experimental data, it is only applicable to empirical scientific inquiry.

The other type of objectivity is based on methods that a scientific community considers to be objective. According to Shala this concerns futures research or foresight because rejecting or accepting hypotheses based on observational and experimental data is not available in this field. According to Shala, effective, i.e., transformative criticism is needed for objectivity in a field like the futures research. While the possibility of criticism does not totally eliminate subjective preference either from an individual's or from a community's practice of futures research, it does provide a means for checking its influence in the formation of scientific knowledge. 
The foci of Kosow's [10] paper are two basic scientific quality criteria of scenarios: consistency and traceability. Traceability of scenarios is often discussed under the terms of transparency, explicitness, accessibility, documentation, and also reproducibility. The reproducibility criterion discussed by Kosow has common and differing features than the informal reproducibility criterion introduced by Amara [3]. As Kuusi will discuss further below, Amara's futures research frame can be seen as a forerunner of the Futures Map approach.

The main contribution of Kosow's paper is the many-sided discussion concerning the consistency criterion. According to Kosow, consistency is a safeguard against arbitrariness of scenarios. It is a substitute for empirical validation, which according to Kosow is not possible and not appropriate with regard to scenarios as their object is not accessible in the present and as they do not claim to be or to become true. As a scenario construction principle, consistency is a kind of heuristic that forces the scenario builder to reflect, how 'bits and pieces' are brought together to form scenarios. According to Kosow, internal consistency refers to the question whether an individual scenario is consistent within itself. Consistency within a scenario sample (or scenario set) refers to the question whether all scenarios of one sample are consistent with one another. The third type of consistency is the consistency between different forms of one scenario, e.g., between a narrative and a numerical form of a scenario. Fourth type is the consistency of underlying models.

In the Futures Map frame, the consistency and traceability criteria belong to the pragmatic external validity criteria of the Futures Map. In our classification, they belong to the group $2 b$.

In summary, the papers of this Topical Collection provide many insights into important aspects of quality criteria. Taken together, they could contribute to establishing the scientific identity of futures research as a discipline in becoming. We hope that the Topical Collection stimulates further discussion concerning quality issues and good practice in futures research.

The background of the collection has been the Futures Map and a set of quality criteria based on it introduced in [14] and [15]. As the other papers of the Topical Collection show, it is possible to define basic concepts of futures research in many other kinds of frames, e.g., in the frames of various versions of the scenario approach. However, we believe that the Futures Map frame is especially useful for the systematic discussion concerning not only basic concepts of futures research but in particular quality criteria of futures research efforts. However, in order to know what 'good foresight' or 'good futures research' really is, how it can be measured and which criteria have to be observed in the beginning and planning phase of a study or project, we still need more discussion, more agreement and joint work on definitions and guidelines.

\section{The Futures Map and its external validity criteria: remarks and illustrations (by Osmo Kuusi)}

\section{Two different interpretations of the future(s) $\operatorname{map}^{1}$}

Many futurists have used the map metaphor when they have discussed alternative pictures of the future or scenarios $[3,5$, $\left.12,{ }^{2} 17,22\right]$.

We can divide the various applications of the map metaphor into two basic categories. For some futurists the future(s) maps are in the minds of people. The maps are their mental models or systems of endosigns [11, 19]. Wendell Bell [3] and Gray Scott [22] use the concept in this way. According to Bell:

As they travel through time, people orient and guide themselves, more or less self-consciously, using their cognitive maps of the future, their hopes and fears. Thus, understanding those maps, even though they are sometimes wildly inaccurate, is essential to explaining people's behavior.

According to Scott, the future map is the mental map of a culture:

Science fiction acts as a philosophical and psychological playground, a place to safely decode what I call the future maps of cultural echoes. These future maps begin in the imagination and psychology of each culture. If we want to visualize these future maps, all we need to do is to listen to the patterns echoing over and over in culture.

The future(s) map of other futures researchers is something concrete like the geographical map. The future(s) map is something that is not just in the mind but like a thing or as a computer file outside the mind. Using the concepts of Kuusi and Hiltunen [13] it is a system of exosigns. The Futures Map of this article introduced in Kuusi et al. [15] is this kind of system of exosigns. Like the geographical map, the Futures Map has many kinds of users or customers. The customers make their own interpretations of future possibilities based on the Map.

A background frame of the Futures Map of Kuusi et al. [15] is the collection of scenarios built using the morphological matrix. Actually, a first draft of the Futures Map can be just a piece of paper with a fixed morphological matrix. Finnish futures researchers use to call it the "Futures Table" (in Finnish tulevaisuustaulu). Using the Futures Table frame, Malaska

\footnotetext{
${ }^{1}$ I use capitals when I speak about a particular version of the future(s) map.

${ }^{2}$ The author of the article used the Finnish word tulevaisuuskartta (the future map) first time already in the 1970s.
} 
and Virtanen [17] gave a mathematic interpretation to the Futures Map related basic concepts.

In the field of business management, Donald Heathfield [5] has had a similar interpretation of his Future Map. He combined the Future Map with the concept of the future "event field". According to Heathfield [5], the "event field" is the picture that appears on the collective "future radar screen". A field of anticipated events could be built for any domain or entity, be it an enterprise or a country (Fig. 1). The goal of the Future Mapping process is to create the event field and then continuously question and clarify this picture using all means available. Like the row variables of the Futures Table, the factors in Heathfield's event field describe the relevant dimensions of the future developments. The rows of the Futures Table have, however, another highly important function. The alternative values of row variables open up a rich view on alternative developments.

The often-used Strategy Map based on balanced scorecards is the third example of a future map based on exosigns. The Strategy Map aims to make the future relevant beliefs of an organization or of its management explicit. In the case of the Strategy Map, the mappers and the customers of the map are the same though typically facilitators from outside the organization participate in the mapping process. Like the customers of the Futures Map of Kuusi et al. [15], the developers of the Strategy Map use the map for the promotion of their vision. According to Kaplan and Norton [8]:

"[S]trategy maps [...] give employees a clear line of sight into how their jobs are linked to the overall objectives of the organization, enabling them to work in a coordinated, collaborative fashion toward the company's desired goals. [...] Strategy implies the movement of an organization from its present position to a desirable but uncertain future position. Because the organization has never been in this future place, the pathway to it consists of a series of linked hypotheses.
A strategy map specifies these cause and effect relationships, which makes them explicit and testable."

The consensus oriented approach seems to be the good choice concerning planning horizon decisions but it seems to be questionable if it is the right strategy, when an organization tries to identify relevant mapping horizon possibilities.

\section{External validity criteria of the Futures Map and Amara's reproducibility criterion}

As is strongly stressed in [15], the basic idea of the validation of the Futures Map is based on validating the whole map. The validation of single or few aspects of the Futures Map is misleading if the impacts on the other aspects of the Map are not taken into account. Only from this starting point the following six pragmatic criteria of the external validity are understandable:

Criterion 1. The number or the scope of possible futures that might be relevant from the point of view of the vision or acceptable futures;

Criterion 2. The most relevant or important possible futures are identified;

Criterion 3. All kinds of causally relevant facts are covered by the identified futures;

Criterion 4. Causally relevant facts are effectively interpreted with as few scenarios as possible;

Criterion 5. Many kinds of users of the Futures Map are able to understand and use it;

Criterion 6. Key customers of the Futures Map are able to understand and benefit from the Map.

An early author who has presented many common ideas with the validation approach of [15b] was Amara [2]. According to Amara:
Fig. 1 "Future radar screen" of the Event field [5]

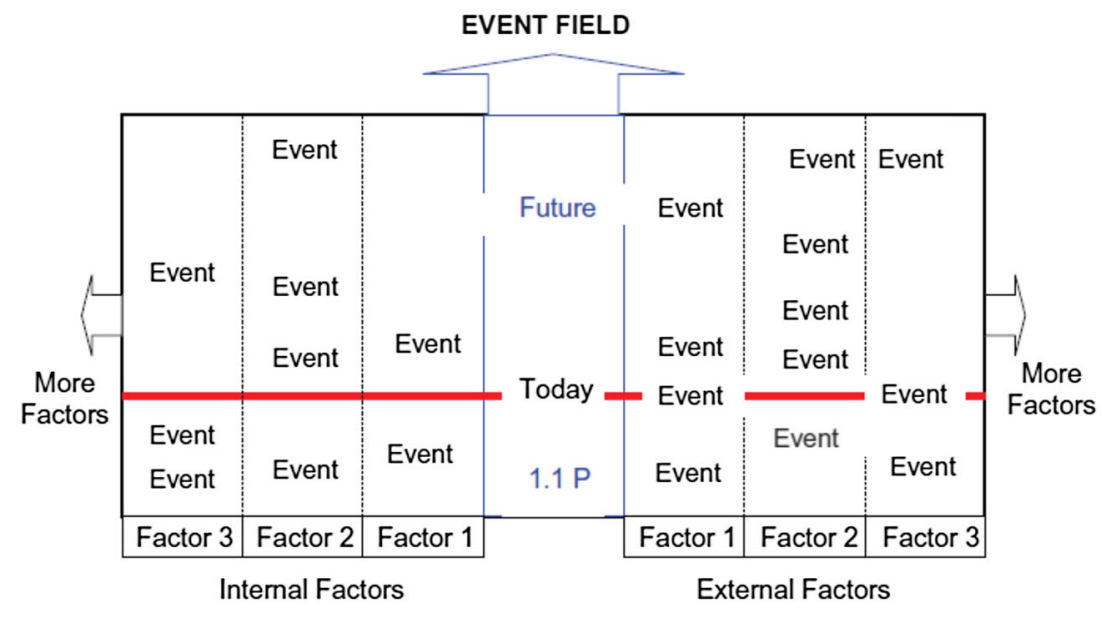


[V]alidation akin to that of scientific prediction (or hypothesis testing) is not appropriate, then what kind is? Validation must be used in the sense of demonstrating that the links between available information and forecasted outcomes are plausible. Plausibility does not mean predictability. The most useful indicator is not necessarily whether the forecast was on target $[\ldots]$ but whether the forecast meets proximate and internal validity criteria.

Though Amara did not use the map metaphor he realized that the validation should be based on the plausibility of various paths of the future. Like in criteria 3 and 4 of the pragmatic external validity above, he considered that the validation should be based on the links between available information and alternative future developments. He introduced the interesting concept of the reproducibility that connects the plausible possible futures and past facts. Amara [2] also nicely discussed the relevance of the futures research effort to its customers, which is the basic feature of the six pragmatic external validity of [15]. Like criterion 1, he also asks: Is the purpose [of futures studies] to provide images of the possible?

For futures research, it has been, however, a great pity that Amara started his article with the thesis: the future is not predictable [2]. I consider that this slogan parroted by many futurists is misleading because it does not take into account both stable and instable elements of the future in the proper way. More suitable thesis is the following: we can predict the future only if we know causal processes that define the future. As is discussed below, this formulation is better in line with Amara's reproducibility criterion as well as with the Futures Map frame of [15].

According to Amara [2], the background of his reproducibility criterion lies in sciences where experimental results are repeatable within a given measurement error. A particular set of inputs operating within a reasonable controlled environment will produce a reproducible set of outputs. Though Amara did not mention it, you can exactly predict the behavioral outputs of many kinds of well-tested systems including some macro systems, e.g., planetary systems. Concerning these systems, the thesis of Amara concerning predictions is misleading.

In order to see the point of the suggested new formulation of Amara's thesis, it is useful to make a distinction between two kinds of systems or beings: not-learning systems or beings and genuine learning systems or beings. These are the two basic categories of the learning based General Theory of Consistency (GTC) paradigm of futures research introduced in [11]. The GTC concludes like Amara that concerning notlearning systems experimental results are repeatable or predictable within a given measurement error. For example, if you include a technological innovation to your scenario, causal processes that determine its behavior in a specified context are typically known. Typically based on its strongly invariant behavioral patterns, you are able to make very precise predictions concerning its behavior. This concerns, however, not the predictions of the future applications or application contexts of the technology. There are also complex causal processes of not-learning systems that you cannot know in practice.

Behavioral sciences have studied stabile behavioral patterns of human beings and other actors. However, unlike the not-learning systems, you can find just transiently invariant causal processes or behavioral patterns in their behavior. Based on learning, actors might change even behavioral patterns that they have without exceptions followed in the past. 'Relevant stakeholders' is the suitable name for actors who are relevant for a specific scenario or for the whole Futures Map. The customers of the Futures Map are one important group of stakeholders and the Futures Map is an instrument of their learning processes.

As Shala [23] has remarked in her article, futures research should be based on the objectivity that differs just from the objectivity based on rejecting or accepting hypotheses concerning invariant behavioral rules of not-learning beings or transiently invariant behavioral rules of learning beings or systems. Shala considers that the alternative objectivity should be based on the "transformative criticism". The idea of the systematic criticism is analogous to the general consistency criterion of the GTC [11]. In practice, the systematic criticism is the effective way to promote the objectivity when expert judgments are used for evidence in Delphi studies. I have suggested that it is highly important to eliminate in any Delphi study the subjective biases of the 'information policy' of the experts [11]. Facilitating many technology Delphi studies, I have learned that the critical comments of other Delphi panelists are decisive in this kind of effort.

The validation of the Futures Map is based on the knowledge concerning relevant causal processes and possible or preferred choices of relevant actors. This conclusion is formulated in [15] as follows:

"Futures researchers typically anticipate the development of complex fields with various interacting causal processes.[...] Theodore Gordon expressed [the second] important starting point of futures research in the following way: 'There is a future without action, and a different one with it. Thus futures research and predestination are, at least on the surface, antithetical'. In other words: people decide on a specific future path by action or inactivity - there is always an alternative."

Amara discussed the stable elements of possible futures using the concept of "informational reproducibility". According to [2], the "informational reproducibility" means that some input of a system results in the same or similar output in various conditions or contexts. In his Consumer Guide, 
Amara [2] suggested three practical criteria of the informational reproducibility that define the probability of an output of a system that gets some input. According to Amara, if the focus of a study is on the probable, the futures researcher should answer the following questions: 1) Is the output repeatable under same conditions? 2) Is the output reproducible under different conditions? 3) Is the output consistent as different assumptions are used?

The Consumer Guide does not make any distinction between not-learning and learning systems. However, you can guess not-learning systems through the criterion that Amara suggests for the evaluation of the possibility of a future image: the image should be within bounds of known physical principles.

Amara considered that the second basic criterion of the possible image of future or scenario is its internal consistency. Kosow [10] has extended the consistency concepts to concern also consistency within a scenario sample (or scenario set), consistency between different forms of one scenario and consistency of underlying models of scenarios.

The consistency concept of the GTC is still more general than the concept of Kosow. Kosow [10] and the GTC share the interpretation that the perception of the consistency is a subjective matter. According to Kosow, scenario storylines rely on holistic consistency 'filters', as intuitive gut feelings, i.e., subjective consistency definitions. In the GTC, the perceived consistency depends on the subjective criteria of sameness of the actor.

The further advantage of the GTC is that it compares the learning (the change in the subjective criteria of sameness) and the perceived inconsistency - for example the unexpected event - to each other. Unlike learning beings or actors, notlearning beings behave consistently following their invariant criteria of sameness. The other basic difference is that Kosow [10] explicitly excludes from the scenario consistency dimensions 'consistency with current knowledge'. For the learning experience, the consistency between the mental model based on the criteria of sameness and the perceived data is decisive.

Amara [2] suggests separate criteria for the evaluation of the probability of futures and for the preferred futures. According to the Consumer Guide of Amara, if the focus is on the preferred futures it is important to study how explicitly the values of authors and the impacts on the stakeholders are portrayed in terms of a) preferences for particular goals; b) tradeoffs between the present and future; c) gains and losses of particular stakeholders.

From the point of view of the Futures Map frame, the criteria of the preferred futures suggested by Amara [2] are useful when the customers of the Futures Map use the map. But like the geographic map, the Futures Map approach separates the Futures Map and its use. The Futures Map does not make any recommendations to its customers. It just shows what kinds of situations of choice they would meet if they would follow any scenario path of the Futures Map. It takes the preferences of its customers into account just in the selection of those scenario paths that it includes to the Futures Map. Surely, the preferences of the mapper and the customers of the Futures Map define these choices. The preferences of the customers are especially important if the focus of the Futures Map is on the external validity criteria 2,4 or 6 . If it has no specified customers (criterion 5) or if the target is to describe various possible (criterion 1) or weak signals based scenarios (criterion 3), the values and preferences of any particular user are not so important.

Of course, the customer of the Futures Map might ask the help of the mapper in the use it. But for example in the Committee for the Future of the Parliament of Finland where the author was permanent adviser and the past prime minister of Finland was the Chair of the Committee, an important choice was to separate the making of the futures research project and the process that resulted in the recommendations made by the Committee. The author was involved in both processes but for the working of the Committee that resulted e.g., in the highly influential suggestion concerning the reform of the Finnish health care system, this separation of the mapping process and the choices based on the Futures Map was important.

\section{Illustrations of the criteria of the pragmatic external validity of the Futures Map}

Kuusi et al. 2015 [15] shortly discusses the content of the six criteria of the pragmatic external validity of the Futures Map. I will next discuss and illustrate the special challenges of futures research processes that are focused on different criteria of the external validity. In my illustrations, I will use the travelling context. Perhaps, this context could be a kind of Caenorhabditis elegans of the discussion concerning the quality criteria of the future(s) map. This small worm has been the highly important model organism in the area of molecular and developmental biology. Travelling or mobility is a nice model context of the Futures Map because:

1) The travelling uses geographical maps. Anybody can easily imagine and understand the mapped future choices related to the travelling.

2) The lengths of planning and mapping horizons of the futures map might vary from 1 day to several decades.

3) The relevant actors might be single persons, various kinds of organizations and regional, national or international planning and decision-making units. Various kinds of independently acting cyborgs (e.g., cars without drivers) seem to be important future actors, too.

4) To anticipate future developments in the context you typically need both quantitative data concerning past choices of actors and the analysis of weak signals or wild cards related, e.g., to technological options. 
5) Recent technological breakthroughs make the context highly important in practical terms. The context is also related to the most challenging wicked problem of the human kind: the climate change.

\section{Futures maps focused on the criteria 1 and 3}

Like the geographical map, the Futures Map is a tool for decision-making of its users. A key challenge of both the geographical map and the Futures Map is the balance between the rich description of the landscape and the simplicity of the map that makes its use easy to the customer(s).

The richness of the Futures Map is the special target of the first and the third criteria of the pragmatic external validity. Using the concept of Ackoff [1], their main function is to avoid the omission error. The omission error is one formulation of the so-called third kind of error in the statistical inference. In the statistical inference, you make the acceptance error if you accept something that you should reject (the type I error). The type II error is the rejection error. You do not accept something that you should accept. In the Futures Map frame, you make the type I or II error if you wrongly either add or reject to add a hypothetical scenario path to the futures map. Using the metaphor of the geographical map, your Futures Map has a type I error if it includes a path that in practice is not available. It has a type II error if your Futures Map does not describe a hypothetical path that is available in the futures landscape.

Ackoff [1] suggested that mistakes of omission are much more serious than the acceptance or the rejection errors. In the futures map frame, the omission error means that you omit possible scenarios or use irrelevant formulations of futures related research questions. Using the geographical map metaphor again, your Futures Map describes for example just paths of areas that you already know quite well. In their methods, futures researchers use to take better into account the omission error than the representatives of other research traditions that are focused on the decision-making practices. Futurists use to understand that if we present wrong or poorly formulated questions concerning different futures we will get irrelevant answers.

If the user has a map that is especially valid in the validity criterion 1, (s)he will less likely meet a future that is not in some way noticed in the map. On the other hand, some scenario and sometimes even most scenarios of the map are irrelevant. The omission error related to the validity criterion 3 means that you have not noticed a scenario that is a real opportunity or treat based on some recent weak signal. On the other hand, a Futures Map that is valid in the criterion 3 often worries about many weak signals that will be irrelevant from the point of view of the customers of the Futures Map.
The consistency requirement discussed by Kosow [10] as well as above concerns also scenarios that are not evaluated from the point of view of relevance, as often is the case in the Futures Maps focused on the criteria 1 or 3. Consistency concerns often the capacities of the relevant actors. If a person has no driving license, to drive a car is not the reasonable option. In the morphological matrix frame, the mapper can make a pairwise consistency evaluation of the alternative characteristics of the key factors related to the scenarios using the scale from 1 (not conceivable, total inconsistent) to 5 (lock and key, perfect match) (compare [4]).

\section{Futures Maps focused on the customer relevance}

A key question concerning the validation of the Futures Map is how to take into account the relevancy aspect. The relevancy is mentioned in all six criteria of the pragmatic external validity and it is especially important concerning criteria 2,4 and 6.

The relevancy of a future possibility means that it is relevant for decision-making: "The Futures Map is first of all the tool for the decision-making of its customers." [15] Besides futures research, many other fields of research have studied practices that are important for decision-making. Decisionmaking options belong to the key research challenges of e.g., economics, management science, psychology and artificial intelligence.

Concerning the knowledge that helps decision-making, futures research can surely learn much more from the results of other research approaches. The mathematical analysis of data is the evident strength of economics or econometrics. The systematic statistical analysis of trends is an important way to make more valid Futures Maps from the point of view of criterion 4. Statistical methods - e.g., regression analysis - are effective ways to analyze the variance of time series of futures relevant variables. Assuming that past trends will continue based on invariant or transiently invariant causal processes, these methods are effective ways to select most relevant scenarios. They also suggest hypotheses concerning causal links between futures relevant variables. However, because relevant actors might change their behavioral patterns, even a good fit between past developments is no guarantee for a valid prediction.

A typical target of a futures research project focused on validity criterion 2 is to find wild cards that are relevant for the customers of the Futures Map. Besides the finding of wild cards, the important task of the mapper is to get the customers to realize their impacts on different stakeholders (criteria 5 and 6). This is actually the feature of a scenario that Kosow [10] called traceability. This is a challenging task because just weak signals without statistical significance use to anticipate most important wild cards. A wild card is often identified based on some "what if"-questions or a trend that is irrelevant from a 
statistical point of view. Only the most innovative experts of the field are able to anticipate emerging development processes and even they are inclined to see the emerging development being realized in the long run. That is why to anticipate of wild cards you need to select experts carefully, who are ready to discuss the developments on the long run. But like Shala [23] warns you should be careful with the "information policies" [11] of experts.

Amara [2] considered that the informational reproducibility or the probability of a scenario is strongest when different individuals using different methods or models produce this scenario. The Strong Prospective Trend (SPT) [18] is a approach, which follows this recommendation. The SPT describes a future development that on the one hand is the continuation of some past trend. On the other hand, an expert panel evaluates that the trend will continue.

The strength of the concept of the SPT is that you can even in quantitative terms make more reasonable probability evaluations of future developments than just using trends based on the past statistics. If actors or representatives of actors are participants of the expert panel you can also take into account their preferences. It is also possible to inform the expert panel about causal links between futures relevant variables based on the past data. In that way, you can really integrate the quantitative past evidence and the qualitative expertise.

A suitable frame for the use of the SPTs is an Argument Delphi study [11]. The crucial feature of the Argument Delphi process is facilitated anonymous argumentation between experts. The size of the panel is $15-40$ persons. The prerequisite of the successful Argument Delphi process is the carefully selected panel. Any panelist should give a special contribution to the study based on his or her special competence and role as the representative of a relevant interest group. The first round of the Argument Delphi is typically based on the interviews of the panelists. The half-structured interviews motivate panelists to invest a lot of effort to the written comments of the next anonymous round.

The trends of futures relevant variables are good a starting point for a Delphi argumentation and judgments concerning the trends. At the end of an argumentation process, you typically have beside SPTs trends concerning which the opinions of experts differ a lot and trends of which most experts do not believe they will continue. In this way, Myllylä [18] studied future prospects of the Murmansk area. His SPTs were related to technological developments, logistic flows, globalization, value changes and demography or living conditions of people. Myllylä's mapping horizon was 2025.

The STPs are especially suitable for futures research studies that are focused on criterion 4. If experts believe on the continuation of some statistically significant trend, you have strong reasons to believe on its continuation. On the other hand, the trends that divide the judgments of the experts concerning their continuation seem to be good sources of possible wild cards. This is a basic idea of the weak signal tool developed by the company Fountain Park [7]. The idea of their tool is that if some experts strongly believe in a specific development and do not change their opinion even if they see the judgments of other experts they often have some special information concerning those things that are relevant for the realization of the possibility. Often these dissidents are not the best experts according to main customers of the Futures Map. They often have expectations or interests that challenge the expectations and recent interests of the key customers (compare [6]).

A common feature of studies that are focused on criterion 4 is that they use information from repetitive choices. If some actors have specified decision rules and if you have evidence concerning the results of their choices you are able to make "good" predictions (forecasts) concerning probabilities of various scenarios. A simple example from the travelling context illustrates this and also the concepts of the planning horizon and the mapping horizon discussed in [15]. It is also a simple illustrating example concerning the fact that you can often make quite precise quantitative forecasts concerning the choices of people.

Let us assume that a person normally uses his car or the train for his way to work. He likes to select the car and if it is not available the train. If these possibilities are not available an emergency solution is to use a bicycle. Let us assume that the evidence from the past shows the probability that the car is not available is 0.1 and the probability is 0.01 concerning the train.

The planning horizon in this case is the next trip to work and the mapping horizon is next trips that the person likes to take into account. The trip with the car is the highly probable business as usual scenario and the train option is an evident other option that belongs to the Futures Map. An interesting question is: how long should the mapping horizon be in order to justify the conclusion that also the bicycle possibility belongs to the Futures Map? The emergency possibility that neither car nor train is available in a choice situation is $0.1 \times 0.01=0.001$. The probability that the bicycle option realizes during 50 decision-making situations is $1-0.999^{50}$ or a bit more than 0.05 . The 0.05 is a typical statistical criterion for the rejection of the 0 -hypothesis. In this case, the 0 -hypothesis is that the bicycle option is not relevant enough for the map. We can conclude that if the mapping horizon is at least next 50 working place visits the person should add the bicycle option to his Futures Map.

\section{Understandable Futures Maps and the avoidance of information overflow: criteria 5 and 6}

Like in the case of a geographic map, the customers of the Futures Map should understand its signs. It is, however, important to realize that a good Futures Map like a good 
geographical map is not just a detailed picture of the "futures landscape', but a working tool for orientation in the futures landscape. A map too detailed of all kinds of possible future paths might be also a too complicated tool for orientation.

First of all, the Futures Map is a tool for orientation of its key customers. The key customers should understand the language or key metaphors of the Futures Map. Sometimes a key customer might even require the use of a 'code language that some other actors do not understand. This is a possible situation concerning the Futures Maps that are focused on criterion 6 but not on criterion 5. On the other hand, if the Futures Map focuses especially on criterion 5 , its basic challenge is to use the language or signs that lay people can understand.

A high quality Futures Map in criterion 6 and especially in criterion 5 avoids the information overflow. How to avoid information overflow? One possibility is not to include into the Futures Map scenarios or pictures of the future that get low probability or informational reproducibility [2] evaluations. But as stressed in validity criteria 1 and 2, this is a questionable choice because some what-if scenarios or scenarios that explain past weak signals might anticipate important wild cards.

A better way to avoid information overflow is to include only those scenarios into the Futures Map that provide information that otherwise may be missed. In other words, you do not evaluate scenarios separately but in the context of all presented scenarios. In order to communicate relevant information with few scenarios, you can use two approaches that complement each other: the principle of the maximal difference and the choice of the most relevant among similar scenarios.

The first step of many scenario processes was the construction of two divergent or contrasting scenarios, assuming that the likely future would be somewhere between the two. This application of the principle of the maximal difference was used already in the famous Shell 1973 scenarios. Having this kind of Futures Map, Shell was prepared for the year 1973 oil crisis. $^{3}$ Generalizing this approach to n-dimensional Futures Table (morphological matrix), you try to cover the ranges of your variables with your contrasting scenarios. If you like to take into account in your Futures Map various what if type scenarios you seldom manage just with two basic contrasting scenarios. However, in order to be understandable to lay people it is seldom reasonable to construct more than four scenarios. If the criterion 5 is the main focus two contrasting scenarios might be enough.

Contrasting scenarios define the basic paths on the Futures Map. The next challenge is to add content to this skeleton of possible future paths. In that effort, the key challenge is to compare related scenarios or possible developments with the

\footnotetext{
$\overline{3 \text { http://s05.static-shell.com/content/dam/shell-new/local/corporate/ }}$ corporate/downloads/pdf/shell-scenarios-40yearsbook080213.pdf
}

closest contrasting scenario(s) and with other scenarios or developments that are close to the same contrasting scenario. Does a new scenario or possible development add relevant content to the Futures Map? Based on this content, should we add the new scenario to the Futures Map? Should we take away another scenario from the Map in order to avoid information overflow?

In futures conferences, the choice between similar scenarios often takes place too early, i.e., before the selection of the contrasting scenarios and helping the selection process. Participants of the conference make first suggestions concerning relevant future developments. For example, they write their suggestions on small pieces of paper. The next step is to collect similar suggestions to clusters. Based on the clusters, facilitators suggest contrasting scenarios discussing with the audience.

In futures conferences, the selection process of relevant scenarios or developments among similar scenarios uses to be an informal process. If the Delphi method with is used, the more explicit or systematic selection process is a thinkable possibility. Concerning the more explicit choice of the most relevant among similar scenarios, the futures researchers can perhaps - benefit from the recent theoretical discussion in the fields of economics and artificial intelligence. Researchers in these fields have suggested three criteria or effects that might be relevant for these kinds of choices: the similarity effect, the attraction effect, and the compromise effect [19, 20]. I will illustrate these effects with examples from the travelling context.

According to [15] if the similarity effect dominates, a new alternative (or an added scenario) decreases the relevance of the other similar scenarios. In the travelling context, let us assume that a contrasting scenario of the Futures Map is a scenario in which commuting will happen using the bicycle instead of the car. The mapper - in this case for example a personal trainer of the traveler - realizes a new possibility of the electric bicycle. Assuming the similarity effect, should the personal trainer keep both scenarios on the Futures Map or just one of them? If the Futures Map discusses on a more general level the future possibilities of healthy travelling (the focus on criterion 5) it would include both alternatives. If the focus is on a specific customer (criterion 6) just one mentioned possibility might be enough.

In the attraction effect, the new scenario is such a relevant choice that two similar scenarios are able to replace or to challenge some earlier contrasting scenario. Let us assume that the traveler has the bicycle but the route from home to the working place requires so much effort that he just seldom uses this option. Let us assume that the personal trainer notices another easier route to the working place besides the electric bicycle. The attraction effect dominates if the reasonable option of the traveler is to use both the electric bicycle and the normal bicycle challenging the contrasting scenario of the car. 
In this case, both types of the bicycle belong to the Futures Map of the traveler.

In the futures map context, we can give the following interpretation to the compromise effect. Two scenarios are combined to a new more relevant scenario. For example, the traveler has sometimes used the bicycle and sometimes walked to the railway station two kilometers away from his home. The personal trainer includes a new option to the Futures Map, in which the traveler uses the bicycle for the walk to the railway station.

Open Access This article is distributed under the terms of the Creative Commons Attribution 4.0 International License (http:// creativecommons.org/licenses/by/4.0/), which permits unrestricted use, distribution, and reproduction in any medium, provided you give appropriate credit to the original author(s) and the source, provide a link to the Creative Commons license, and indicate if changes were made.

\section{References}

1. Ackoff RL (2006) A major mistake that managers make http:// www.acasa.upenn.edu/A_MAJOR_MISTAKE.pdf Accessed 21 November 2015

2. Amara R (1981) How to tell good work from bad. Futurist XV(2): 63-71

3. Bell W (2002) Making people responsible: the possible, the probable, and the preferable. In: Dator JA (ed) Advancing futures: futures studies in higher education. Praeger Studies on the 21st Century, Westport, pp 33-52

4. Grienitz V, Hausicke M, Schmidt AM (2014) Scenario development without probabilities - focusing on most important scenario. Eur J Futur Res 15:27

5. Heathfield D (2007) Building a comprehensive strategic future management system: a futures map approach. In: Sharpe B, van der Heijden K (Ed.) Scenarios for Success: Turning Insights in to Action. Wiley

6. Hiltunen E (2013) Foresight and innovation. How companies are coping with the future? Palgrave, 2013

7. Ilmola L, Kuusi O (2006) Filters of weak signals hinder foresight: monitoring weak signals efficiently in corporate decision-making. Futures 38(8):908-924
8. Kaplan RS, Norton DR (2000) Having trouble with your strategy? Then map it, harvard business review

9. Kononiuk A, Sacio-Szymańska A (2015) Assessing the maturity level of foresight in Polish companies - a regional perspective. Eur J Futur Res

10. Kosow H (2015) New outlooks in traceability and consistency of integrated scenarios. Eur J Futur Res

11. Kuusi O (1999) Expertise in the future use of generic technologies, government institute for economic research, research reports 59, Doctoral thesis, Helsinki, J-Paino Oy

12. Kuusi O, Kamppinen M, Rubin A, Malaska P (2003) Tulevaisuuden tekeminen (The Making of Future). In: Kamppinen M, Kuusi O, Söderlund S (eds) Tulevaisuudentutkimus (Futures Research). SKS, Helsinki

13. Kuusi O, Hiltunen E (2011) The signification process of the future sign. J Futur Stud 16(1):47-66

14. Kuusi O, Cuhls K, Steinmüller KH (2015) Quality criteria for scientific futures research. Futura 1

15. Kuusi O, Cuhls K, Steinmüller KH (2015) The futures map and its quality criteria. Eur J Futur Res

16. Longino HE (1990) Science as social knowledge: values and objectivity in scientific inquiry, 4. print. Princeton University Press, Princeton

17. Malaska P, Virtanen I (2009) Theory of Futuribles and Historibles. Futura 1:65-84

18. Myllylä Y (2007) Murmanskin alueen teollinen, logistinen ja sosiaalinen kehitys vuoteen 2025 (The Industrial, Logistic and Social Development of the Murmansk Area until 2025) Doctoral dissertation, University of Joensuu

19. Osogami T, Otsuka M (2014) Restricted Boltzmann machines modeling human Choice, http://papers.nips.cc/paper/5280restricted-boltzmann-machines-modeling-human-choice.pdf Accessed 21 November 2015

20. Rieskamp JR, Busemeyer R, Mellers BA (2006) Extending the bounds of rationality: evidence and theories of preferential choice. J Econ Lit 44:631-661

21. Rohrbeck R, Schwartz O (2013) The value contribution of strategic foresight: insights from an empirical study of large European companies. Technol Forecast Soc Chang 80:1593-1606

22. Scott G (2014) Visualizing the Future, http://www.wfs.org/futurist/ 2014-issues-futurist/july-august-2014-vol-48-no-4/visualizingfuture Accessed 21 November 2015

23. Shala E (2015) A new path in foresight validation? Discussing the socio-epistemic underpinning of quality criteria. Eur J Futur Res

24. Vasamo AL (2015) Radical Technology Inquirer (RTI) Tool for technology anticipation and evaluation: introduction and quality criteria analysis. Eur J Futur Res 